REGARDS

SUR LEECONOMIE ALLEMANDE

BULLETIN ECONOMIQUE DU CIRAC
Regards sur l'économie allemande

Bulletin économique du CIRAC

105 | 2012

Varia

\title{
France-Allemagne : une entente a minima
}

\section{René Lasserre}

\section{OpenEdition}

Journals

Édition électronique

URL : http://journals.openedition.org/rea/4415

DOI : $10.4000 /$ rea. 4415

ISBN : 978-2-8218-1420-2

ISSN : 1965-0787

Éditeur

CIRAC

Édition imprimée

Date de publication : 11 juillet 2012

Pagination : 1-2

ISSN : 1156-8992

Référence électronique

René Lasserre, «France-Allemagne : une entente a minima », Regards sur l'économie allemande [En ligne], 105 | juillet 2012, mis en ligne le 19 juillet 2012, consulté le 22 septembre 2020. URL : http:// journals.openedition.org/rea/4415; DOI : https://doi.org/10.4000/rea.4415 


\section{Foncièrement robuste, malgré des risques croissants}

Dans l'UE, l'économie allemande se distingue par sa robustesse foncière. Elle tire sa force exportatrice essentiellement de la compétitivité hors-prix d'une industrie qui a su adapter son offre de produits comme sa gamme de prix à l'évolution de la demande sur un marché où flux et process sont largement globalisés. Patiemment reconquise dans un singulier effort collectif de réformes et ajustements successifs durant ces vingt dernières années, sa compétitivité globale est entretenue depuis par un marché du travail flexible, un système de protection sociale dont le financement est assuré à terme, et des finances publiques en cours de consolidation. Mais son insertion réussie au sein du marché communautaire comme dans le partage mondial du travail expose l'Allemagne, plus que les autres Etats de l'UE, aux risques conjoncturels extérieurs, et tout particulièrement à l'évolution imprévisible de la crise de la dette souveraine dans la zone Euro. De ce fait, soulignent tous les experts, les prévisions sont difficiles en ce moment, et les scénarii de croissance varient beaucoup.

Le peu de lisibilité incite les acteurs économiques à la prudence : en juin comme en mai, l'indice indice ifo a reculé. Certes, les entreprises industrielles jugent toujours favorablement leur situation actuelle, mais elles sont réservées quant aux perspectives des mois à venir. Deux secteurs font exception : le commerce de détail et le BTP, qui ont renoué avec l'optimisme en juin. Le climat des affaires reflète fidèlement l'évolution d'une conjoncture qui voit faiblir légèrement un moteur des exportations qui n'en reste pas moins puissant (en 2011, l'excédant commercial était monté à 158,1 milliards $€$ ), et se confirmer l'essor que prend depuis un an la consommation intérieure.

Au premier trimestre, l'excédent commercial a contribué comme toujours à tirer la croissance : elle a été de 0,5\%,compensant largement le léger recul enregistré au précédent (-0,2\% ; Destatis). La structure géographique des exportations s'est modifiée, sous l'impact du tassement de l'activité au sein de la zone Euro: la demande en provenance de cette dernière a reculé. A l'inverse, effet d'une conjoncture mondiale porteuse, les commandes issues des pays tiers ont confirmé leur tendance à la hausse. La production industrielle est restée stable. Certes, l'industrie ayant engrangé moins de gros contrats qu'en mars, les commandes ont reculé de près de $2 \%$ en avril (Bundesbank, rapport de juin), sauf en ce qui concerne les biens d'investissement $(+0,75 \%)$. Alors que celles en provenance de la zone Euro ont diminué $(-2,25 \%)$, celles des pays tiers progressaient $(+1 \%)$, de même que les commandes domestiques $(+1,25 \%)$. Si la production industrielle a reculé par rapport à mars $(-2,25 \%)$, c'est du fait de circonstances exceptionnelles : les statistiques sur la construction mécanique étaient gonflées par l'arrivée tardive des données communiquées par les entreprises, le 'pont' du $1^{\text {er }}$ mai a réduit le nombre de jours ouvrés, de même que les grèves d'avertissement dans le cadre des négociations salariales dans la métallurgie. Au total, l'activité industrielle semble avoir surmonté le léger creux du $1^{\mathrm{er}}$ trimestre. Quant au BTP, il profite du retour d'une météo plus clémente, mais aussi de ce qu'en ces temps incertains, les ménages préfèrent investir dans la pierre.

La demande intérieure reste bien orientée. Par rapport au dernier trimestre 2011, les dépenses publiques et privées (respectivement $+0,2 \%$ et $+0,4 \%$ ) étaient en hausse au premier, compensant le recul des investissements dans les biens d'équipement et la construction $(-0,8 \%$ et $-1,3 \%$; Destatis). Cela dit, la dynamique apparaît plus nettement en comparaison annuelle: les dépenses publiques et privées ont progressé respectivement de $1,9 \%$ et $1,8 \%$; quant aux investissements dans les biens d'équipement, ils étaient en hausse de $2,5 \%$, signe de la bonne tenue de l'activité industrielle. Seul le BTP enregistre un léger recul $(-0,5 \%)$, dû surtout à la fin des programmes de soutien à la conjoncture adoptés en 2009.

L'indice GfK confirme que les ménages gardent le moral malgré la crise de la dette souveraine. Certes, les risques externes pesant sur l'économie allemande les angoissent, mais cela n'entame pas leur confiance quant à leur situation personnelle et l'évolution de leurs revenus. Plusieurs constats les incitent à garder le moral. En mai, l'inflation est retombée en-dessous du seuil 'psychologique' des $2 \%$, ce qui a un effet
Une croissance tirée par deux moteurs

L'activité industrielle se maintient

Les consommateurs gardent confiance 
Taux de chômage : 55 \%

L'Allemagne recrute dans l'UE

Rigueur et croissance vont de pair positif sur la perception du pouvoir d'achat. A la suite des négociations salariales dans plusieurs branches importantes, ils ont vu leur revenu disponible augmenter de 3,6\% au premier trimestre, un 'coup de pouce' aussitôt dépensé pour l'acquisition de biens durables ou l'achat de logements (Destatis), car le taux d'épargne (11\%) n'a guère varié. La consommation des ménages tire aujourd'hui elle aussi très nettement la croissance, contribuant à enrayer la baisse du potentiel de croissance qui s'observait en Allemagne depuis les années 1970, souligne la Bundesbank (rapport d'avril).

L'optimisme des ménages résulte également de la bonne tenue du marché de l'emploi. Le nombre de chômeurs inscrits est tombé à 2,8 millions en juin, soit un taux de chômage de 6,6 \% selon l'Agence fédérale pour l'emploi (juin), ou de 5,5 \% (2,32 millions de personnes) selon les critères de l'OIT (mai ; Destatis). Le nombre de chômeurs indemnisés au titre de l'assurance chômage s'élevait à 768000 (+ 26000 seulement en un an; Agence de Nuremberg). En revanche, celui des bénéficiaires de l'assistance chômage (" Hartz IV »), bien qu'en baisse (97 000 de moins), s'élève toujours à près de 2 millions. Quant au nombre d'emplois vacants, il a enregistré une légère hausse pour se situer à 499000 , et il vise surtout des profils qualifiés dans les secteurs phares de l'industrie, ou la logistique et la santé. Le nombre d'actifs occupés a fortement augmenté en un an (+561000 personnes) pour atteindre 41,58 millions (Destatis), la plus forte hausse étant celle des emplois soumis à cotisations sociales, qui sont aujourd'hui 28,88 millions (+ 666000 ; Agence de Nuremberg). Fait à relever : cette augmentation concerne tous les Länder et tous les secteurs, mais est particulièrement prononcée dans l'industrie et les services aux entreprises.

Cela étant, cette évolution tend désormais à ralentir, pour des raisons conjoncturelles, et bien plus encore structurelles. Après avoir été divisé par deux depuis son niveau historique de 2005 , le chômage ne devrait plus guère reculer maintenant. La réserve latente a été fortement mobilisée ; elle a diminué de moitié depuis 2006 et compte aujourd'hui un peu moins de 4 millions de personnes. Seuls les plus de 60 ans offrent encore un potentiel inexploité de même que, fait nouveau, l'immigration au sein d'une UE où la mobilité des actifs s'est accrue. 280000 actifs étaient venus s'installer en Allemagne en 2011, ils occupent essentiellement des emplois soumis à cotisations sociales ; quelque 200000 sont attendus cette année et l'an prochain. C'est ainsi que le gouvernement fédéral compte résoudre le manque de main-d'œuvre qualifiée généré par le vieillissement démographique: en recrutant massivement les compétences au sein du marché communautaire (via par exemple la plateforme make-it-ingermany.com), et en mobilisant mieux encore la réserve latente, tout en misant de manière plus systématique sur l'activation des chômeurs grâce à une offensive de formation afin de garantir le haut niveau de qualification des ressources qui est l'un des pivots de la compétitivité globale du site.

Le cap de consolidation des finances publiques mené par les gouvernements du Bund et des Länder contribue de manière essentielle à entretenir la confiance des acteurs économiques. Le gouvernement fédéral, qui vient d'adopter le projet de loi budgétaire 2013 et le programme 2012-2016 (ils seront soumis au parlement à partir du 11 septembre), compte respecter la 'règle d'or' à son niveau (déficit structurel limité à $0,35 \%$ du PIB) dès 2013 et renoncer à tout nouvel endettement dès 2016. Cette rigueur n'exclut nullement une politique de croissance: I'investissement, notamment en matière d'infrastructures et de formation comme de recherche, sera maintenu bon an mal an aux alentours de 25 milliards $€$.

" UN BUDGET INTELLIGEMMENT ASSAINI crée la confiance sans laquelle consommation et investissement sont inconcevables ", rappelait récemment W. Schäuble dans une tribune libre (Les Echos, 14-05-2012). La Bundesbank prolonge cette analyse, rappelant l'importance qu'ont eue assainissement des finances et réformes structurelles sur le marché du travail et dans le domaine de la protection sociale pour permettre à une économie au potentiel de croissance bridé de renouer avec la compétitivité (rapport d'avril). C'est cette politique qui a rendu l'Allemagne plus résistante aux crises exogènes et lui confère aujourd'hui la fonction "d'ancre de stabilité " dans une zone Euro en proie à la crise de la dette souveraine. Ce qui la fragilise actuellement, plus que les risques de chocs exogènes, c'est l'incertitude qui plane quant à la volonté de ses partenaires de se plier à la même discipline européenne.

(I. Bourgeois, 04-07-2012) 